Journal of Fluorescence, Vol. 7, No. 1, 1997

\title{
Frequency-Domain Fluorescence Lifetime Imaging for Endoscopic Clinical Cancer Photodetection: Apparatus Design and Preliminary Results
}

\author{
G. Wagnières, ${ }^{1,2}$ J. Mizeret, ${ }^{1}$ A. Studzinski, ${ }^{1}$ and H. van den Bergh ${ }^{1}$
}

Received June 14, 1996; accepted October 25, 1996.

\begin{abstract}
We describe a new fluorescence imaging device for clinical cancer photodetection in hollow organs in which the tumor/normal tissue contrast is derived from the fluorescence lifetime of endogenous or exogenous fluorochromes. This fluorescence lifetime contrast gives information about the physicochemical properties of the environment which are different between normal and certain diseased tissues. The excitation light from a $\mathrm{CW}$ laser is modulated in amplitude at a radio frequency by an electrooptical modulator and delivered by an optical fiber through an endoscope to the hollow organ. The image of the tissue collected by the endoscope is separated in two spectral windows, one being the backscattered excitation light and the other the fluorescence of the fluorochrome. Each image is then focused on the photocathode of image intensifiers (II) whose optical gain is modulated at the same frequency as the excitation intensity, resulting in homodyne phase-sensitive images. By acquiring stationary phase-sensitive frames at different phases between the excitation and the detection, it is possible to calculate in quasi-real time the apparent fluorescence lifetime of the corresponding tissue region for each pixel. A result obtained by investigating the endogenous fluorochromes present in the mucous membrane of an excised human bladder is presented to illustrate this method and most of the optical parameters which are of major importance for this photodetection modality have been evaluated.
\end{abstract}

KEY WORDS: Light-induced fluorescence; frequency domain; photodetection; cancer; fluorescence lifetime imaging; endoscopy; optical biopsy.

\section{INTRODUCTION}

Because an important portion of the advanced-stage cancers is difficult to treat effectively, it is important to detect them at an early stage. (1) Many invasive cancers are preceded by alterations, such as dysplasia, or carcinoma in situ (CIS). ${ }^{(2,3)}$ However, for most of these early lesions, there are no effective methods to detect these stages of the disease. Most cancers originate in superfi-

\footnotetext{
' Institute of Environmental Engineering, Swiss Federal Institute of Technology (EPFL), CH-1015 Lausanne, Switzeriand.

${ }^{2}$ To whom correspondence should be addressed at LPAS, Bâtiment de Chimie, EPFL, CH-1015 Lausanne, Switzerland.
}

cial tissues (skin cancer) or in the tissues lining the body cavities or tubes such as bronchial cancers of the lung and cancers of the esophagus, stomach, colon, bladder, and female reproductive organs. Furthermore, the use of light-induced fluorescence (LIF) spectroscopy originated with the development of medical endoscopes by numerous investigators as a potentially powerful method of detecting and localizing malignant tumors.

Tissue characterization using LIF spectroscopy can be divided into two categories. The first is based on the detection of the native tissue fluorescence (autofluorescence), which induces an optical contrast between the lesion and the surrounding normal tissues. Among other things, tissues are made up of proteins, nucleic acids, 
lipids, and water with fluorescing and nonfluorescing chromophores. Many tissues are known to fluoresce in the UV and visible part of the spectrum. ${ }^{(4,5)}$ The groups of Alfano, ${ }^{(6)}$ Lohmann, ${ }^{(7)}$ and Yuanlong ${ }^{(8)}$ conducted pioneering studies in this field which, to some extent, gave rise to the increasing number of papers on the autofluorescence excitation and emission spectra of human and animal tissues performed in vitro and in vivo.(9,15)

The second approach concerning the clinical use of LIF spectroscopy for detecting and localizing malignant tumors is based on the administration of a fluorescent drug such as hematoporphyrin derivative (HPD) or its somewhat purified form Photofrin II, ${ }^{(16,23)} \delta$-aminolevulinic acid-induced protoporphyrin IX (ALA-PPIX), ${ }^{(24,27)}$ tetra(meta-hydroxyphenyl)chlorin (mTHPC), ${ }^{(28)}$ and fluorochromes coupled to monoclonal antibodies. ${ }^{(29)}$ Such "drugs" tend to localize to some extent selectively in certain tumors after intravenous injection. The region where a superficial cancer is suspected is then irradiated with light at a wavelength at which more dye fluorescence is induced from the tumor, with its relatively high drug concentration, than from the dye-deficient surrounding normal tissue.

The interest in studying the time-resolved fluorescence spectroscopy of exogenous and endogenous fluorochromes to characterize biological tissues is connected with two major reasons.

(1) The optical contrast between the lesion and the surrounding normal tissue is a key parameter limiting the performance of cancer LIF photodetection. This contrast is unfortunately degraded frequently when a steady-state LIF technique is used due to, among other things, tissue autofluorescence in both tumor and normal tissue. As the above-mentioned clinically used exogenous dyes have fluorescence lifetimes which are different from that of the endogenous fluorochromes, better discrimination (improved contrast) between these two dyes can be obtained in principle using time-resolved fluorescence imaging.

(2) Several endogenous and exogenous fluorochromes are more or less sensitive to their local environment. According to the literature, some microenvironmental parameters differ between normal tissues and some neoplastic lesions. Among these, one might mention the $\mathrm{pH},{ }^{(30)}$ the DNA and/or RNA content, ${ }^{(31)}$ the cell membrane permeability, which can influence the access of the fluorochrome to certain microcompartments such as the nucleus, ${ }^{(32,33)}$ and the enzymatic activity. ${ }^{\left({ }^{34,35)}\right.}$ As the fluores- cence lifetime is very sensitive to small perturbations in the environment of the fluorochrome, it is of interest to evaluate the performance of time-resolved LIF spectroscopy for tissue characterization based on the physicochemical properties of the local environment. More important is the fact that, in principle, environmentally sensitive dyes do not require selective localization in a tumor for effective marking.

Fluorescence lifetimes can be determined from the inverse slope of the exponential decay of the light intensity following pulsed excitation. This is the so-called "time-domain" approach. The corresponding approach for the imaging of fluorescence lifetime consists of pixelby-pixel scanning and time-correlated single-photon counting. ${ }^{(36)}$ Such measurements require a considerable amount of time, and it may not be practical to produce high-resolution and/or real-time images. Other laboratories have created time-decay contrasts using time gating of the long-lived fluorescence. ${ }^{\left({ }^{37.38}\right)}$ However, these methods provide information only on the presence of the delayed emission. Moreover, on-gating of delayed emission does not appear to have contrast opportunities such as particularly lifetime-based contrast and selective observation of either short or long components of the fluorescence decay. Obviously, the technique of pulsed image intensifier gating is restricted to fluorescence lifetimes that differ by at least one order of magnitude to allow for an efficient lifetime-based selective signal suppression.

Another approach, which avoids most of the above mentioned drawbacks of the time-domain approach, consists of using "frequency-domain" fluorescence lifetime imaging (FLIM) techniques as described below.

One of the novelties of our implementation of this method is that fluorescence and reference images are taken simultaneously and treated in quasi-real time. The main advantage of such simultaneous measurement compared to sequential detection is that exactly the same area is imaged in the different spectral bands independently of the scene movements. Moreover, the device described below allows for an immediate observation of the endoscopic area under white light illumination during fluorescence detection to localize the origin of the fluorescence signals of interest.

\section{EXPERIMENTAL}

\section{Operating Principle of the FLIM Apparatus}

The basic principle of "frequency-domain" FLIM has been described by Lakowicz et al. ${ }^{(39,41)}$ This group 


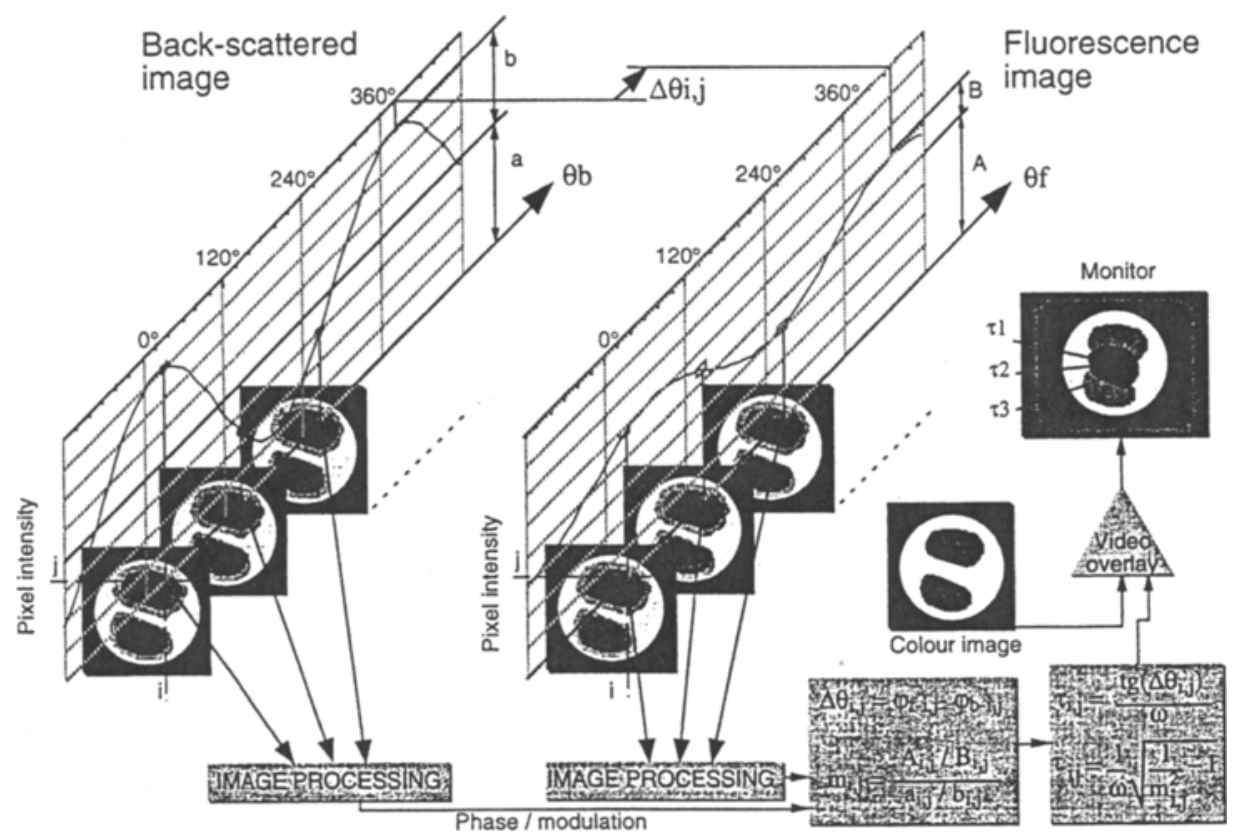

Fig. 1. Principle and signal processing of the frequency-domain fluorescence lifetime imaging apparatus. Three reference (backscattering) and three fluorescence images are detected simultaneously for three different phases from the fluorescence excitation. Detection of this type enables determination of the phase and modulation of both images for each pixel. The fluorescence lifetime is then deduced from the phase difference and the modulation ratio for each pixel at the video frequency.

essentially applied this technique in the context of fluorescence microscopy experiments.

Briefly, the principle of this approach consists of measuring the fluorescence lifetimes or decay times $\tau$ by the phase method, in which the sample is illuminated with fluorescence excitation light modulated at a radio frequency $\omega$. The basic idea is to observe the fluorescence image with an image intensifier (II), whose optical gain is modulated at the same frequency, resulting in homodyne phase-sensitive images. Detection of this type will preserve the decay time information, as the twodimensional output signal at the position $\vec{r}$ in the image plane depends on the phase angle difference between the fluorescence emission and the gain modulation of the II.

The phase shift $\Delta \theta$ between the excitation light and the fluorescence and/or the modulation ratio $M[M=$ (AC/DC components of the fluorescence)/(AC/DC components of the excitation)] enables (see Fig. 1) the value of this lifetime in the case of a monoexponential decay to be obtained. These two parameters are related to the fuorescence lifetime by

$$
\begin{aligned}
& \tau(\vec{r})=\tan \frac{\Delta \theta(\vec{r})}{\omega} \\
& \tau(\vec{r})=\frac{1}{\omega} \sqrt{\frac{1}{M^{2}(\vec{r})}-1}
\end{aligned}
$$

In the case of multiexponential decays, the values of $\tau$ obtained with Eqs. (1) and (2) are generally not identical.

It is not possible to determine a fluorescence lifetime from a single phase-sensitive image. Various approaches, based on the detection of fluorescence images at two or more phase shifts, have been proposed by Lakowicz et al. to measure the fluorescence lifetime. ${ }^{(39)}$

The approach we are using consists of determining the phase angle and/or the modulation at each point in the image by examining the detector phase-angle dependence of the image intensity for three phase shifts of the gain-modulation signal. This approach reduces several artifacts generated by the methods based on the detection at two phases only.

As presented in Fig. 1, two gain-modulated image intensifiers are used in our experimental setup. One of them (II 1) detects the tissue fluorescence, whereas the other one (II 2) detects the backscattered fluorescence excitation light. The reference value of these phase angle $\left(\theta_{R}\right)$ and modulation $\left(m_{R}\right)$ are obtained with II 2 , whereas II 1 measures the fluorescence phase $\left(\theta_{\mathrm{F}}\right)$ and modulation $\left(m_{\mathrm{F}}\right)$. These stationary phase-sensitive images are collected using two CCD cameras, digitized, and manipulated with a mathematical operator in real time (quasi-video frequency) to determine the absolute phase 
and modulation of each pixel. A second mathematical operator calculates the phase difference $(\Delta \theta)$ and/or modulation ratio $(M)$ between the two images series, as well as the value of $\tau$.

The resulting image therefore consists of pixels whose value corresponds to the fluorescence lifetime only. Hence, for "small" modulation frequencies (less than $5 \mathrm{GHz}$ ), the intensity of this lifetime image no longer depends on the fluorochrome concentration, excitation light intensity (which is quite heterogeneous in an endoscopic context), tissue-endoscope distance, and fluorochrome photobleaching (as long as the photoproducts are not fluorescing). This lifetime image is finally combined with the corresponding color image obtained during white-light illumination of the endoscopic field.

An advantage of the experimental method proposed here is that pixel-to-pixel scanning is not required to obtain the fluorescence lifetime image, as the information from all pixels is obtained at the same time by the CCD.

\section{Design and Characterization of the FLIM Apparatus}

The major factor degrading the endoscopic detectability of small tumors is either low signal levels resulting in high image noise or insufficient contrast between tumor and surrounding normal tissue. Thus contrast, noise and, to a lesser extent, image sharpness are key parameters in early cancer detection using a fluorescence imaging technique. The typical surface of an early-stage squamous cell carcinoma in the tracheobronchial tree, the esophagus, or the upper aerodigestive tract is of the order of $1 \mathrm{~cm}^{2}$ at the time of detection. ${ }^{(23)}$ Thus, at a typical observation distance, such a lesion occupies a significant portion of the field of view. The resolution of most standard medical endoscopes, image intensifiers, and $\mathrm{CCD}$ cameras is therefore sufficient for detecting such lesions. Therefore, when designing such an apparatus, the focus is on the reduction of noise and contrast enhancement of the tumor image.

Noise is due mainly to photon statistics (shot noise) in the context of such experiments as discussed below. This noise can therefore be reduced by increasing the number of detected photons. For a given apparatus, detection geometry, tissue optics, and tumor marker, this can be done either by increasing the fluorescence excitation light intensity or by increasing the detection period. The former is limited by the dye photobleaching and the latter by loss of image sharpness when moving the endoscope. Other factors that might limit the use of high light intensity are thermal effects in the tissue.
Under our conditions, the excitation light intensity is typically of the order of $50 \mathrm{~mW} / \mathrm{cm}^{2}$ at the tissue surface. Increasing the detection period to decrease the noise and/or to perform the calculation between the different images is limited by the movement of the scene, i.e., integrating or calculation periods that are too long would cause the image to streak and hence its quality to decrease. The frequency of intensity fluctuations corresponding to the maximum of the human eye temporal contrast sensitivity is between 5 and $10 \mathrm{~Hz}$ at the luminosity of conventional TV monitors. ${ }^{(42)}$ Thus we found that the upper limit for time integration is of the order of $200 \mathrm{~ms}$ for a lifetime-reconstructed image, which corresponds to five consecutive PAL video frames.

\section{The Endoscopes}

In general we use standard medical rigid (Storz) and flexible (Olympus) endoscopes. Unfortunately, such endoscopes are not optimal for cancer photodetection, as the effective diameter of the objective which captures the light is small (typical diameter, $300 \mu \mathrm{m}$ ). This small diameter is due to the presence of biopsy, inflation, and/or suction channels and to their broad (typically $75^{\circ}$ ) field of view. Typically, if $20 \mathrm{~mm}$ is between the distal end of the endoscope and the cancer, the probability for the light emitted by the tissue to enter the objective is around $10^{-4}$ ! Furthermore, the imaging light guide fiuoresces when excitation light is reflected back to the objective from the surface being inspected. Thus, at particular surface orientations, a strong specular reflection may induce degradation of both the fluorescence and the reference images.

For the time being, we have designed our apparatus to be compatible with most standard endoscopes available which deliver images of widely varying diameters and with different apertures.

\section{Light Sources and Illumination Optics}

The fluorescence excitation light source presented in Fig. 2 is a continuous-wave argon ion pumped dye laser system (Coherent, Palo Alto, CA; Innova $100+$ CR599). Such a tunable device provides more than $500 \mathrm{~mW}$ between 390 and $950 \mathrm{~nm}$ if different spectral configurations (dyes, mirrors) are used. Moreover, the argon ion laser itself can be used in the "mid-UV" (334-, 351-, 364-nm) or "deep-UV" (275-, 305-nm) modes if shorter wavelengths are required. This laser beam is then modulated in intensity at a radio frequency by a tunable (1- to $350-\mathrm{MHz})$ Pockels cell (Quantum Technology, Lake Mary, FL; 


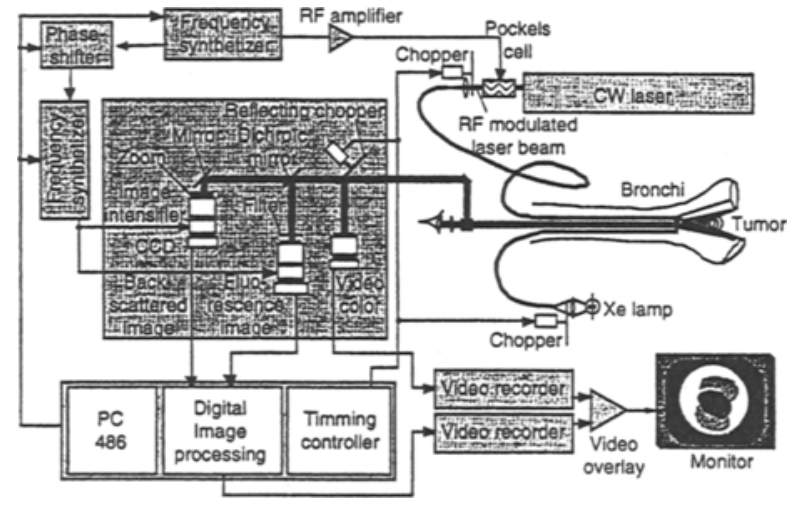

Fig. 2. Experimental setup of the frequency-domain FLIM apparatus for endoscopic tissue characterization.

300- to 700-nm coated KDP crystal) combined with a polarizer. This Pockels cell is operated by an amplified (Quantum Technology; Type 3500 amplifier) frequency generator (Hewlett Packard HP 8648B).

Obturation of the modulated laser beam is achieved by a rotating optical chopper controlled by a central clock. Such an obturation is required by the fact that our apparatus alternates between two modes of observation. The first one consists of illuminating the endoscopic scene with white light and acquiring the normal endoscopic color image. In the second mode, laser excitation only is performed and the fluorescence and backscattered images are recorded simultaneously at three phases (separated by $120^{\circ}$ between 0 and $360^{\circ}$ ) from the laser modulation. In this second mode, the laser beam is coupled into a $200-\mu \mathrm{m}$-core diameter quartz optical fiber to illuminate the endoscopic site.

The white-light source of our apparatus is a $300-\mathrm{W}$ xenon lamp (Storz, Tuttlingen, Germany; Type 487B). An optical chopper controlled by the central timing controller switches off this white light during observation of the endoscopic field in the fluorescence mode. The transition time is about $2 \mathrm{~ms}$, which enables alternation between these two modes at video frequency. The light provided by this white-light source is then coupled into the illumination optics of the endoscope.

\section{Detection Optics, Spectral Design, and Detectors}

The dye fluorescence, excitation backscattered light, and tissue autofluorescence in one mode and the color image in the other mode are collected by the endoscope objective. At the ocular end of the endoscope a beamsplitter cube is used to transmit $10 \%$ of the light for visual observation of the white-light image (cf. Fig.
2). The other $90 \%$ is reflected toward a Storz articulated optical arm which is quite flexible and transmits $50 \%$ at $630 \mathrm{~nm}$. It consists in five rigid segments with Hopkins lenses. The quality of the image transmitted is superior to that of a fiberscope. The latter has a typical transmission of $15 \%$ at this wavelength. The ocular at the end of the articulated arm is inserted into a black box which contains further optics, both gain-modulated image intensifiers, and the JVC (Model TK $1270 \mathrm{E}$ ) video color CCD camera. This ocular furnishes a divergent beam which varies from one endoscope to another and attains at most $15^{\circ}$, with a pupil of maximum $2-\mathrm{mm}$ diameter. A photographic lens (Nikon $f=50 \mathrm{~mm}$; aperture, 1.8) images this pupil on the reflecting chopper, which either lets the fluorescent and backscattered images pass or reflects the white-light image toward the video color camera. This chopper is also controlled by the central clock. The focusing on the reflective chopper is necessary to keep the dead time to a minimum $(\sim 1 \mathrm{~ms})$ between the two modes. A dichroic mirror separates the spectral domains corresponding to the dye fluorescence and the excitation light. Another bandpass filter is placed in front of the image intensifier, which amplifies the dye fluorescence image, whereas a narrow bandpass filter is placed in front of the other image intensifier to detect the backscattered excitation light only.

The zoom lenses (Fujinon H6X11.5B-MDM21) are necessary to make the apparatus compatible with many types of endoscopes. For instance, the size of the endoscopic image may vary by as much as a factor of 3 according to the type of endoscope used. More importantly, however, if one decreases the size of an image on the intensifier, the intensity per pixel increases, and the signal-to-noise ratio is improved. The consequent loss of resolution can be tolerated easily under these conditions. Proximity focused image intensifiers are the only electrooptical devices which can be used in the context of these experiments due to the weak fluorescence yield of the fluorochromes investigated, the small collection efficiency of the endoscope objective, and the necessity to use imaging detectors the optical gain of which can be modulated at radio frequencies. These two-stage, fastgated image intensifiers (Hamamatsu V5548MOD) have a typical maximum gain of 15,000 candela $/ \mathrm{m}^{2} / \mathrm{lux}$. Their "multialkali" photocathode is sensitive between 180 and $820 \mathrm{~nm}$. The output of their phosphorescent screen (Type P20; $\lambda_{\text {MAX }}=550 \mathrm{~nm}$ ) is coupled to a $[739(\mathrm{H})$ $\times 509$ (V)-pixel] black-and-white CCD camera (Hamamatsu C 5985-10) with relay lenses (Hamamatsu A2098). The modulation of the optical gain of the image intensifiers is performed by changing the voltage between their photocathodes and the input of their micro- 


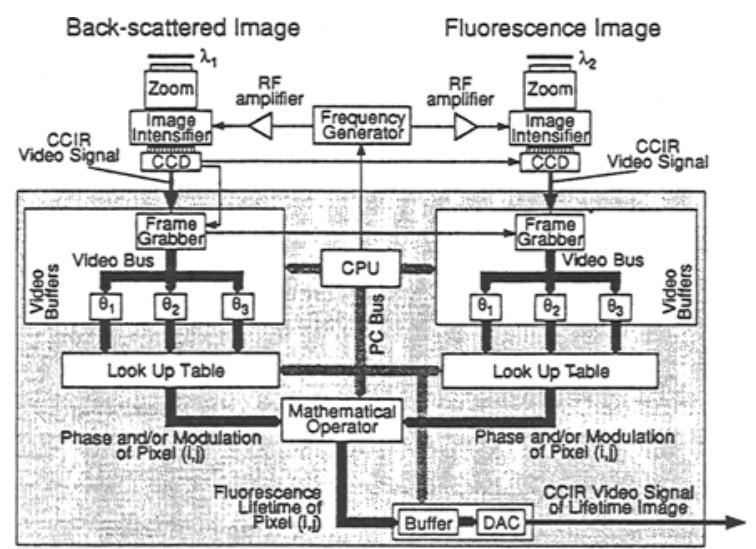

Fig. 3. Electronic diagram of the frequency-domain FLIM apparatus.

channel plate. This voltage is controlled by a frequency generator (Hewlett Packard HP 8657B) which is "phase-locked" with the frequency generator controlling the Pockels cell. A 12-bit phase shifter (Merrimac, West Caldwell, NJ; PLB-3B-10B) controlled by the computer is placed on the $10-\mathrm{MHz}$ reference signal to change the phase of the HP $8657 \mathrm{~B}$ frequency generator (see Fig. 2).

The overall transmission at $630 \mathrm{~nm}$ for this optical setup is $20 \%$ if a Storz $0^{\circ}$ esophagoscope is used. This transmission concerns all the optical components located between the endoscope objective and the photocathode of the image intensifier.

\section{Signal Processing and Electronic Design}

The acquisition and signal processing of the fluorescence and backscattered images are performed separately (Fig. 3). An image is recorded by the CCD for each phase shift (three in our case) between the fluorescence excitation and the II gain modulation as presented in Fig. 1. The integration time for each of these frames can be set from $40 \mathrm{~ms}$ to $1 \mathrm{~s}$. The images are then transferred to a frame grabber as a standard video signal (Comité Consultatif International de Radio-communication, CCIR). After digitization ( 8 bits) in the frame grabber, the images are stored in a video memory (buffer). These buffers (three per channel) are refreshed sequentially. Therefore, one given memory always stores an image acquired with the same phase shift. After each acquisition, the three buffers are scanned simultaneously. For each pixel, the 24 bits ( $3 \times 8$ bits) are transferred to a fast programmable "fitting" operator (look-up table) which contains preprogrammed computed values of the phase and/or modulation. As the two channels are synchronized, the output of the two lookup tables concern the same pixel at a given time. These two values are then transferred to the mathematical operator ( $2 \times 8$-bit look-up table). It calculates the apparent fluorescence lifetime of the corresponding pixels from these two values and stores the result in a buffer. For calculation of the fluorescence lifetime based on the phase shift, the operation consists in subtracting the phase of the reference image from the fluorescence image and applying expression (1). Similarly, the fluorescence lifetime can also be obtained from the modulation. It consists of calculating the relative demodulation (Fig. 1 ) and applying formula (2). Once the whole image is completed, it is converted back to an analog video signal (CCIR) and, finally, overlaid on the color image (Fig. 2). In addition, both the result and the color images can be stored digitally on a hard disk (PC or external) or analogically on video recorders.

The principal difficulty is the synchronization between all electronic devices and the mechanical components (laser, white light, and reflecting choppers). This synchronization is based on the video signal (clock of one of the CCD) for the analog part of the circuitry (including the analog-to-digital conversion) and by the CPU for the digital part. One of the CCD, that of the backscattered image channel as in Fig. 3, synchronizes the other so that the two images are recorded at the same time. The same synchronization signal is used by the frame grabbers. When the image is fully digitized, the CPU transfers it to the proper video buffer. During the acquisition of the next frame, the CPU scans the content of the $3+3$ buffers. The outputs of the look-up tables are updated for each new address in 25 ns (static-RAM). The CPU triggers the beginning of the analog conversion to a CCIR signal when the stored image in the output buffer is completed. The three choppers are also controlled by the CPU via a timing controller card which also controls the phase shifter.

\section{RESULTS AND DISCUSSION}

Our FLIM apparatus was initially evaluated in vitro using diluted fluorochromes with known fluorescence lifetimes from the literature. ${ }^{(43)}$ Rhodamine $6 \mathrm{G}(3 \mu \mathrm{g} / \mathrm{ml}$, $\left.\tau=6 \mathrm{~ns}, \lambda_{\mathrm{EM}}=560 \mathrm{~nm}\right)$ and acridine orange $(10 \mu \mathrm{g} / \mathrm{ml}$, $\tau=2 \mathrm{~ns}, \lambda_{\mathrm{EM}}=530 \mathrm{~nm}$ ) were dissolved in water for this purpose and their fluorescence was induced with green $(514 \mathrm{~nm})$ light of the argon ion laser modulated at $64 \mathrm{MHz}$. The lifetimes measured with our apparatus using both the phase shift and the modulation change 


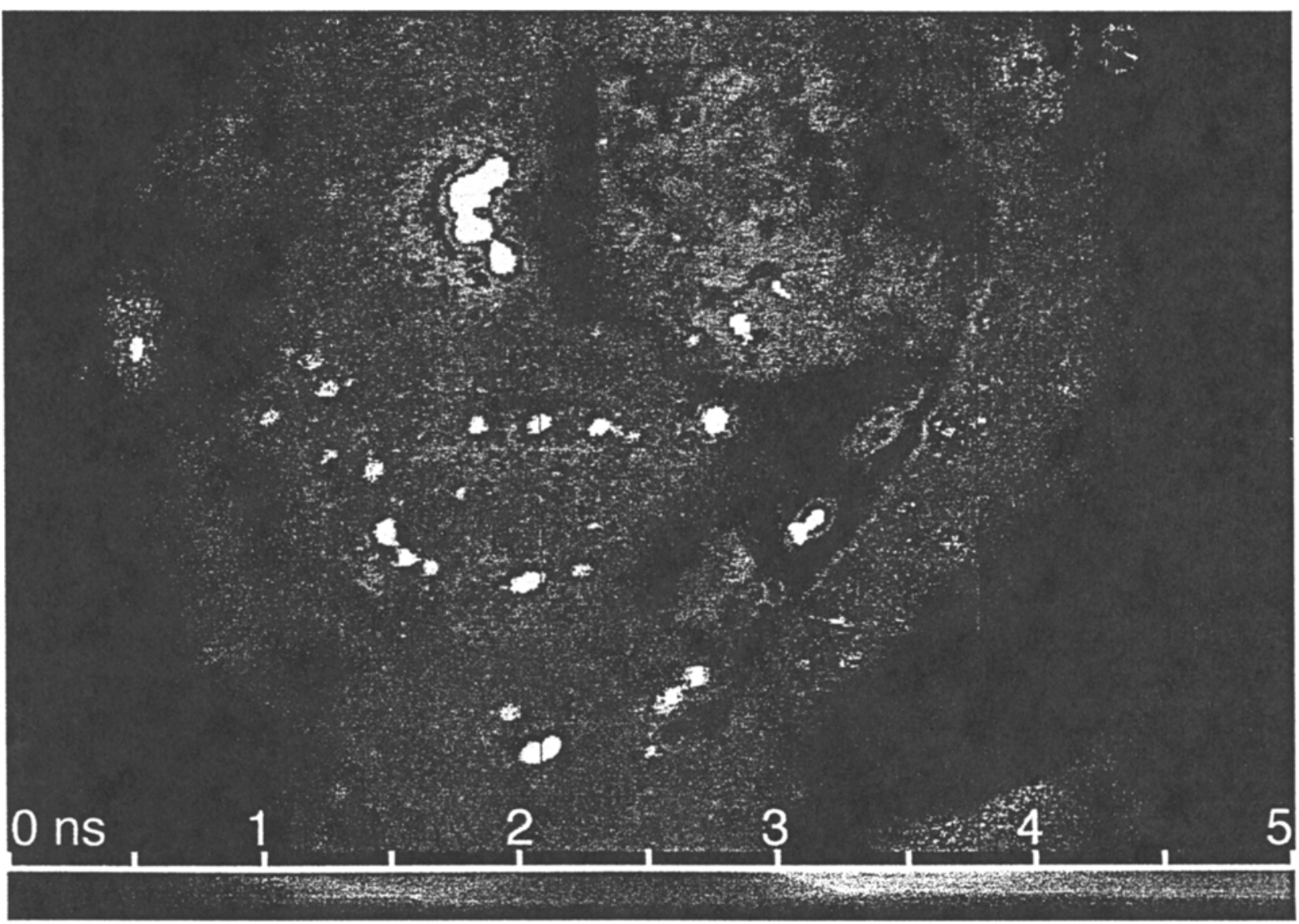

Fig. 4. Fluorescence lifetime image obtained with an opened, excised human bladder. The color of the pixel indicates the fluorescence lifetime, from $0 \mathrm{~ns}$ (deep blue) to $5 \mathrm{~ns}$ (red). The area located in the upper right comer fluoresces with a lifetime of about $0.5 \mathrm{~ns}$ (blue), whereas the lower left area exhibits a longer lifetime of about $2.5 \mathrm{~ns}$ (green). The complete tissue sample has been characterized by serial histological sectioning. The short lifetime corresponded to ulceration of the mucous membrane, whereas the other part was normal urothelium. See the text for more information concerning the detection conditions.

were consistent with the predicted values of these lifetimes.

Preliminary, on-bench experiments were performed on a freshly excised human bladder. This bladder was opened after resection and examined by our FLIM apparatus. The fluorescence of the endogenous fluorochromes was also induced with green (514-nm) light. This wavelength, according to the literature, corresponds to the absorption of molecules of the flavin molecule family. ${ }^{(4,5)}$ The illumination intensity of this tissue sample was $1 \mathrm{~mW} / \mathrm{cm}^{2}$. The filter used to reject the fluorescence excitation light was a longpass $\lambda>550-\mathrm{nm}$ filter and the modulation frequency was $40 \mathrm{MHz}$. Figure 4 shows the image of the effective fluorescence lifetime as measured on the basis of the phase-shift. The pixel hue gives the fluorescence lifetime. The area located in the upper right corner fluoresces with a lifetime of about 0.5 ns (blue area in Fig. 4), whereas the lower left area ex- hibits a longer lifetime of about 2.5 ns (green area in Fig. 4). The complete tissue sample has been characterized by serial histological sectioning. The short lifetime corresponded to ulceration of the mucous membrane, whereas the other part was normal urothelium. Also, the short fluorescence lifetime corresponded to a strong fluorescence emission. Several specular reflections which induced saturation are clearly visible in this lifetime image (white spots). These saturations were detected by the reference camera. It is unfortunately impossible to eliminate such artifacts using a standard optical filtration method. One approach consists of assuming that the phase and modulation reference, usually given by the reference image, is uniform over the entire field of view. This assumption is valid as long as $\Delta d<<2 \pi c / \omega$, where $c$ is the velocity of light and $\Delta d$ is the maximal distance difference between the distal end of the endoscope and various points of the field of view. Under our 
conditions, this method is feasible as long as the modulation frequency $\nu=\omega / 2 \pi$ is smaller than typically 50 $\mathrm{MHz}$. Hence, the investigation of fluorochromes with a lifetime $>5$ ns using the FLIM apparatus can be performed with one image intensifier only, the reference being detected by a simple "point" detector.

It can be shown that the limitation of such imaging devices based on the tissue autofluorescence is essentially due to the shot noise. It is not a straightforward matter to determine the optimal approach which would enable the effect of this noise on the final result (i.e., the value of the fluorescence lifetime) to be minimized. Various approaches, based on the detection of fluorescence frames at two or more phases, have been proposed by Lakowicz et al. to induce a contrast based on the fluorescence lifetime. ${ }^{(39)}$ It has been suggested that the minimum number of frames necessary to resolve any monoexponential decay is three, which is consistent with the fact that the number of parameters to be determined is also three (i.e., the average amplitude, modulation, and phase; the frequency is known). With noise, the sine wave calculation is not unique, i.e., different acquisition of the same fluorescence will lead to sets of different lifetimes. The number of frames has to be chosen to minimize this distribution. On the one hand, if the number of frames is increased, the fitting is more precise (increase of the redundancy). On the other hand, increasing the number of frames will reduce the fluorescence acquisition time per frame and therefore will decrease the signal-to-noise ratio. The results of an analytical study and computer simulation presented elsewhere ${ }^{(44)}$ have shown that the effect of the shot noise on fluorescence lifetime images is not dependent on the number of frames for a given illumination intensity and total acquisition time. This result is of major importance, as it indicates that lifetime recovery is possible and optimal from three 8-bit images processed in real time using a look-up table or a fast operator.

The study described in the above-mentioned reference has also shown that, for a given excitation frequency, the effect of the shot noise on the lifetime image is smaller with phase shift- than with demodulationbased measurements for fluorescence lifetimes which are smaller than $1 / \omega$. For fluorescence lifetimes which are longer than $1 / \omega$, the situation is inverted, i.e., demodulation-based measurements give better results. These simulation and calculation results were verified for different modulation frequencies with the tissue samples described above (results not shown here).

These tests on a freshly excised human bladder enabled us to evaluate the optical parameters specific to this type of photodetection. As the spectral CCD detec- tor sensitivity $\left(0.15 \mathrm{~mJ} / \mathrm{cm}^{2}\right.$ per frame at $550 \mathrm{~nm}$ to obtain saturation), the CCD accumulation time $(100 \mathrm{~ms})$ per frame and the typical gain of the image intensifiers $\left(10^{3} \mathrm{~W} / \mathrm{cm}^{2} / \mathrm{W} / \mathrm{cm}^{2}\right)$ were known, it is possible to determine the illumination of the fluorescence image intensifier's photocathode. This illumination, due to the tissue autofluorescence, is of the order of $10^{-8} \mathrm{~W} / \mathrm{cm}^{2}$ if the normal bladder wall is observed from a distance of 20 $\mathrm{mm}$ under typical green light illumination intensity (10 $\mathrm{mW} / \mathrm{cm}^{2}$ at $\left.514 \mathrm{~nm}\right)$. Such illumination of the photocathode corresponds to 10,000 photons or to 1000 photoelectrons per pixel and per frame (photocathode quantum efficiency $\cong 10 \%$ ). Under these conditions, we calculated that the resulting standard deviation is about $1 \mathrm{~ns}$ for a lifetime of $5 \mathrm{~ns}$ measured at $32 \mathrm{MHz}$.

As the diameter of the endoscopic image on the image intensifier's photocathode $(3 \mathrm{~mm})$, the tissue illumination $\left(10 \mathrm{~mW} / \mathrm{cm}^{2}\right)$, the overall transmission of the optics $(20 \%)$, and the diameter and the field of view of the endoscope's objective $\left(300 \mu \mathrm{m}\right.$ and $75^{\circ}$, respectively) are known, the tissue autofluorescence yield can be evaluated. This estimate leads to the value of $10^{-3}$ autofluorescence photons emitted by the tissue per excitation photon at $514 \mathrm{~nm}$.

In conclusion, our preliminary study showed that a tissue differentiation can be performed on the basis of tissue autofluorescence only using a fluorescence lifetime imaging technique. Moreover, we have shown that such endoscopic tissue characterization can be performed in quasi-real time (several refreshes per second) by detecting the optimal number of three phase-resolved fluorescence images.

The experience gained in this study will help in the design of FLIM apparatuses for endoscopic diagnosis.

\section{ACKNOWLEDGMENTS}

The authors are grateful to the Fonds National Suisse de la Recherche Scientifique, the Programme prioritaire optique, the CHUV-EPFL-UNIL fund, CibaGeigy, the Swiss Cancer League, the Vaudoise, Valaisanne and Genevoise Cancer Leagues, the Fondation Dreyfuss (Lausanne), and the Fondation E. Muschamp for financial support.

\section{REFERENCES}

1. P. C. Norwell (1986) Cancer Res. 46, 2203-2207.

2. R. Pasche, M. Savary, and Ph. Monnier (1981) Acta Endosc. 11, 9277-291. 
3. T. Muto, J. R. Buessey, and B. C. Morson (1975) Cancer 36, $2251-2270$.

4. S. Undenfriend (1962) Fluorescence Assay in Biology and Medicine, Vol. I, Academic Press, New York.

5. G. M. Brenboin, A. N. Domanskii, and K. K. Turoverov (1969) Luminescence of Biopolymers in Cells, Plenum Press, New York.

6. R. R. Alfano, D. B. Tata, J. J. Cordero, et al. (1984) IEEE J. Quant. Electr. 20, 1507-1511.

7. W. Lohmann (1988) Naturwissenschaften 75, 201-202.

8. Y. Yuanlong, Y. Yanmin, L. Furning, et al. (1987) Lasers Surg. Med. 7, 528-532.

9. D. Braichotte, G. Wagnières, Ph. Monnier, et al. (1991) SPIE $1525,211-218$.

10. R. R. Alfano, A. Pradhan, and G. C. Tang (1989) J. Opt. Soc. Am. B 6(5), 1015-1023.

11. P. N. Yashke, R. F. Bonner, P. Cohen, et al. (1989) Gastrointest. Endosc. 35, 184

12. R. Marchesini, M. Brambilla, E. Pigoli, et al. (1992) J. Photochem. Photobiol. B Biol. 14, 219-230.

13. K. T. Schomacker, J. K. Frisoli, C. C. Compton, et al. (1992) Lasers Surg. Med. 12, 63-78.

14. W. S. Poon, K. T. Schomaker, T. F. Deutsch, et al. (1992) J. Neurosurg. 76, 679-686.

15. R. Richards-Kortum, R. P. Rava, R. E. Petras, et al. (1991) Photochem. Photobiol. 53, 777-786.

16. R. C. Benson, G. M. Farrow, J. H. Kinsey, et al. (1982) Mayo Clin. Proc. 57, 548-555.

17. G. Wagnières, Ch. Depeursinge, Ph. Monnier, et al. (1990) SPIE $1203,43-52$.

18. A. E. Profio, O. J. Balchum, and F. Cartens (1986) Med. Phys. $13,717-721$

19. T. Hirano, M. Ishizuka, K. Suzuki, et al. (1989) Lasers Life Sci. 3(2), 99-116.

20. R. Baumgartner, H. Fisslinger, D. Jocham, et al. (1987) Photochem. Photobiol. 46(5), 759-763.

21. S. Montan, K. Svanberg, and S. Svanberg (1985) Opt. Lett. 10 , 56-58.

22. G. Wagnières, D. Braichotte, A. Chatelain, et al. (1991) SPIE 1525, 219-236.

23. Ph. Monnier, M. Savary, Ch. Fontolliet, et al. (1990) Lasers Med. Sci. 5, 149-169.
24. J. C. Kennedy and R. H. Pottier (1992) J. Photochem. Photobiol. 14, 275-292.

25. M. Forrer, Th. Glanzmann, J. Mizeret, et al. (1995) SPIE 2324, $84-88$

26. P. Jichlinski, H. -J. Leisinger, M. Forrer, et al. (1997) Lasers Surg. Med. (in press).

27. M. Kriegmair, R. Baumgartner, R. Knuechel, et al. (1994) Urology 44(6), 836-841.

28. D. Braichotte, J. -F. Savary, G. Wagnières, et al. (1994) SPIE 2081, 62-73.

29. S. Folli, G. Wagnières, A. Pèlegrin, et al. (1992) Proc. Natl. Acad. Sci. USA 89, 7973-7977.

30. S. Mordon, V. Maunoury, J. -M. Devoiselle, et al. (1992) J. Photochem. Photobiol. B Biol. 13, 307-314.

31. H. S. Rye, S. Yue, D. E. Wemmer, et al. (1992) Nucleic Acids Res. 20, 2803-2812.

32. P. Herlin, J. Mamay, J. H. Jacob, et al. (1983) Endoscopy 15, 4 7.

33. O. Kocher, M. Amaudruz, A. M. Schindler, et al. (1981) J. Submicrosc. Cytol. 13, 267-281.

34. L. Massaad, I. de Waziers, V. Ribrag, et al. (1993) Bull. Cancer 80(5), 397-407.

35. G. Weber (1976) Advances in Enzyme Regulation, Vol. 15, Pergamon Press, New York.

36. S. F. Wang, S. Kitajima, T. Uchida, et al. (1990) Appl. Spectrosc. 44(1), 25-30.

37. W. L. Rumsey, J. M. Vanderkooi, and D. F. Wilson (1988) Science 241, 1649-165 I.

38. M. Kohl, U. Sukowski, and B. Ebert (1993) SPIE 1881, 206-221.

39. J. R. Lakowicz and K. W. Berndt (1991) Rev. Sci. Instrum. 62(7), 1727-1734.

40. J. R. Lakowicz, H. Szmacinski, K. Nowaczyk, et al. (1992) Anal. Biochem. 202, 316-330.

4I. J. R. Lakowicz, H. Szmacinski, K. Nowaczyk, et al. (1992) Proc. Natl. Acad. Sci. USA 89, 1271-1275.

42. D. E. Pearson (1975) Transmission and Display of Pictorial Information, Pentech, London.

43. F. W. D. Rost (1991) Quantitative Fluorescence Microscopy, Cambridge University Press, Cambridge.

44. J. Mizeret, G. Wagnières, A. Studzinski, et al. (1995) SPIE 2627, $40-48$. 